\title{
Development of Power Electronics Based Test Platform for Characterization and Testing of Magnetocaloric Materials
}

\author{
Deepak Elamalayil Soman, Jelena Loncarski, Lisa Gerdin, Petter Eklund, \\ Sandra Eriksson, and Mats Leijon \\ Department of Engineering Sciences, Ångström Laboratory, Uppsala University, Lägerhyddsvägen 1, P.O. Box 534, \\ 75121 Uppsala, Sweden
}

Correspondence should be addressed to Deepak Elamalayil Soman; deepak.soman@angstrom.uu.se

Received 12 September 2014; Revised 15 December 2014; Accepted 31 December 2014

Academic Editor: Gorazd Stumberger

Copyright (C) 2015 Deepak Elamalayil Soman et al. This is an open access article distributed under the Creative Commons Attribution License, which permits unrestricted use, distribution, and reproduction in any medium, provided the original work is properly cited.

\begin{abstract}
Magnetocaloric effects of various materials are getting more and more interesting for the future, as they can significantly contribute towards improving the efficiency of many energy intensive applications such as refrigeration, heating, and air conditioning. Accurate characterization of magnetocaloric effects, exhibited by various materials, is an important process for further studies and development of the suitable magnetocaloric heating and cooling solutions. The conventional test facilities have plenty of limitations, as they focus only on the thermodynamic side and use magnetic machines with moving bed of magnetocaloric material or magnet. In this work an entirely new approach for characterization of the magnetocaloric materials is presented, with the main focus on a flexible and efficient power electronic based excitation and a completely static test platform. It can generate a periodically varying magnetic field using superposition of an ac and a dc magnetic field. The scale down prototype uses a customized single phase $\mathrm{H}-$ bridge inverter with essential protections and an electromagnet load as actuator. The preliminary simulation and experimental results show good agreement and support the usage of the power electronic test platform for characterizing magnetocaloric materials.
\end{abstract}

\section{Introduction}

In 1976 it was proved by Brown that the magnetocaloric effect could be used for substantial cooling effect near room temperature [1]. Magnetic refrigeration has two main advantages: it can reach higher efficiency compared to conventional techniques and there is no need for refrigerant gases [2]. The efficiency of magnetic refrigeration can reach $30 \%-60 \%$ of the Carnot cycle, whereas vapor compression refrigeration reaches only $5 \%-10 \%$ of the Carnot cycle [3].

When a magnetocaloric material is subjected to an external magnetic field the magnetic dipoles of the atoms align, causing a reduction in the spin-entropy. If this is done in an adiabatic manner, the entropy of the lattice must increase with an increase in the material's temperature. In a cooling cycle this heat is removed. When the magnetic field is removed, thermal entropy transforms into magnetic entropy, which results in cooling of the material. Hence heat is transferred from the surroundings to the material. This phenomenon can be used in the construction of refrigerators $[4,5]$ and the materials usually considered for such applications are referred to as magnetocaloric materials.

The most important efforts made in experimental work during the last decades are summarized in a few review papers $[1,3,6,7]$. The magnetocaloric effect is determined by the entropy change, $\Delta S$, if the magnetic field is changed isothermally, and by the adiabatic temperature change, $\Delta T_{\mathrm{ad}}$, if the magnetic field is changed adiabatically. These two parameters are not directly coupled, which is why they have to be optimized simultaneously. When investigating magnetic refrigeration a suitable value for the magnetic flux density is around $1 \mathrm{~T}[2]$.

There are two generations of magnetic refrigerators and heat pumps according to $\mathrm{Yu}$ et al. The first generation's machines use superconducting magnets and operate at 
a low frequency. The breaking point between the two generations came with a machine using permanent magnets and a rotating disc of magnetocaloric materials, which was constructed by Astronautics Corporation of America [3]. All magnetic machines in both first and second generation are devices containing either moving beds of magnetocaloric material, which enter and exit a static magnetic field, or magnets moving over a static bed of magnetocaloric material $[3,6]$. According to $\mathrm{Yu}$ et al. periodic movements, linear or rotational, are required in order to obtain continuous powers of a magnetic refrigerator or heat pump. All researchers inventing these magnetic machines have faced more or less the same problems regarding design and operation of the machines [3].

This work shows the preliminary and entirely new approach of combining the rather conventional power electronics with essential control and the novel idea to employ a specific magnetic circuit for the testing of the magnetocaloric materials. In particular, the generation of the periodically varying magnetic flux density is obtained using superposition of an ac magnetic field and a dc magnetic field. The focus of most previous research projects within magnetic refrigeration has been on the thermodynamic part, whereas, in this paper, focus lies on the possibilities of using power electronics in order to obtain a varying magnetic flux density. To obtain a varying magnetic flux density a simple conventional power electronics setup with additional protection has been employed to control a very unusual and purely inductive load. The main benefit of using power electronics is that both magnet and magnetocaloric material can be stationary. Furthermore, density and frequency of the magnetic field can easily be changed by the control algorithm.

Preliminary measurements and simulations of a scaled down prototype of magnetocaloric setup, which can generate a biased alternating magnetic field for the purpose of testing of magnetocaloric materials in terms of testing the magnetocaloric properties on a small sample material, have been the main focus of this paper. The ac magnetic field is generated by an electromagnet, which is powered by an external dc-source connected to a single phase $\mathrm{H}$-bridge inverter. The dc magnetic field is generated by a permanent magnet, connected to the electromagnet in a magnetic circuit according to Figure 1. Hence, the magnetic circuit consists of an E-formation iron core with a coil and a permanent magnet placed on the outer branches and an $8 \mathrm{~mm}$ air gap on the middle branch. The ac and dc magnetic field are superposed, creating a biased magnetic field in the air gap.

A review and comparison of the efficiency of different magnetic devices states that use of the permanent magnets is more efficient compared to the use of electromagnets or superconducting electromagnets [7]. Since no device combining a permanent magnet and an electromagnet has been constructed before $[3,7]$, it is not possible to say how efficient such a device would be in relation to existing devices. An electromagnet requires power to produce a magnetic field, which is why a permanent magnet is preferable [7]. However, the device investigated in this paper does not need a motor in order to magnetize and demagnetize the magnetocaloric

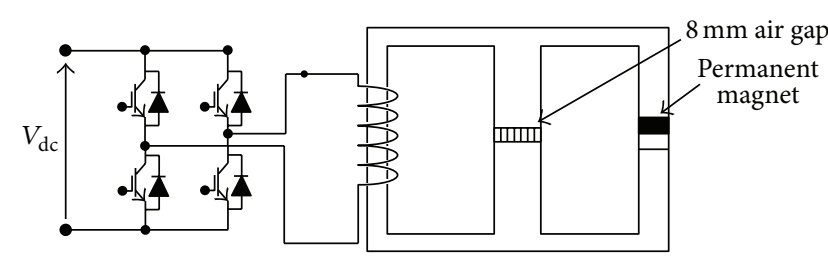

FIGURE 1: Simple representation of the test platform.

material, which might open up for new ways of designing efficient magnetic refrigerators or heat pumps.

\section{Power Electronics and Protection}

Experimental setups used for the study of magnetocaloric materials are mostly based on rotating disc type mechanisms, where the induced flux density frequency depends on the speed of the rotating disc. These arrangements need complex control mechanisms to obtain the desired speed simultaneously as the desired flux. A power converter based flux induction method is more compact and is able to provide easier control for magnetization and demagnetization of the material.

In this section, the power converter system used for the magnetocaloric experiment is described. A voltage source inverter (VSI) is used for powering up the electromagnetic coil. The VSI converts dc power to corresponding ac at the desired frequency.

Due to the requirements of the experimental setup the inverter has to be able to operate in a low frequency area (around $5 \mathrm{~Hz}$ ) and manage a purely inductive load. The frequency of the magnetic field should be variable in order to enable investigation of how magnetocaloric materials react at different time scales; hence the operating frequency of the inverter has to be variable as well.

The power converter has to be designed to satisfy all the requirements of the electromagnet and at the same time it should be able to cope with the highly inductive load stresses. As the operating frequency can vary from very low to high during testing all the protections should work during the entire range of operation. The custom made inverter thus has four main stages: accurate gate drive circuit with optical isolation, insulated-gate bipolar transistor (IGBT) based $\mathrm{H}$ bridge power stage, load current limit circuit, and Labview CompactRIO based field programmable gate array (FPGA) digital controller.

2.1. IGBT Gate Drive Circuit. The gate drive circuit of the inverter has been customized to satisfy all the operating requirements. Primary protection features are also included in this part. The complete circuit representation of the gate driver is shown in Figure 2. Optically isolated gate driver IC HCPL-316J from Avago is selected as the core component for the circuit [8]. Soft IGBT turn off, Desat protection, and under voltage lock-out (UVLO) protection features are ensured by this IC. Moreover the optical isolation of PWM pulses and fault feedback signal are incorporated in the chip. 


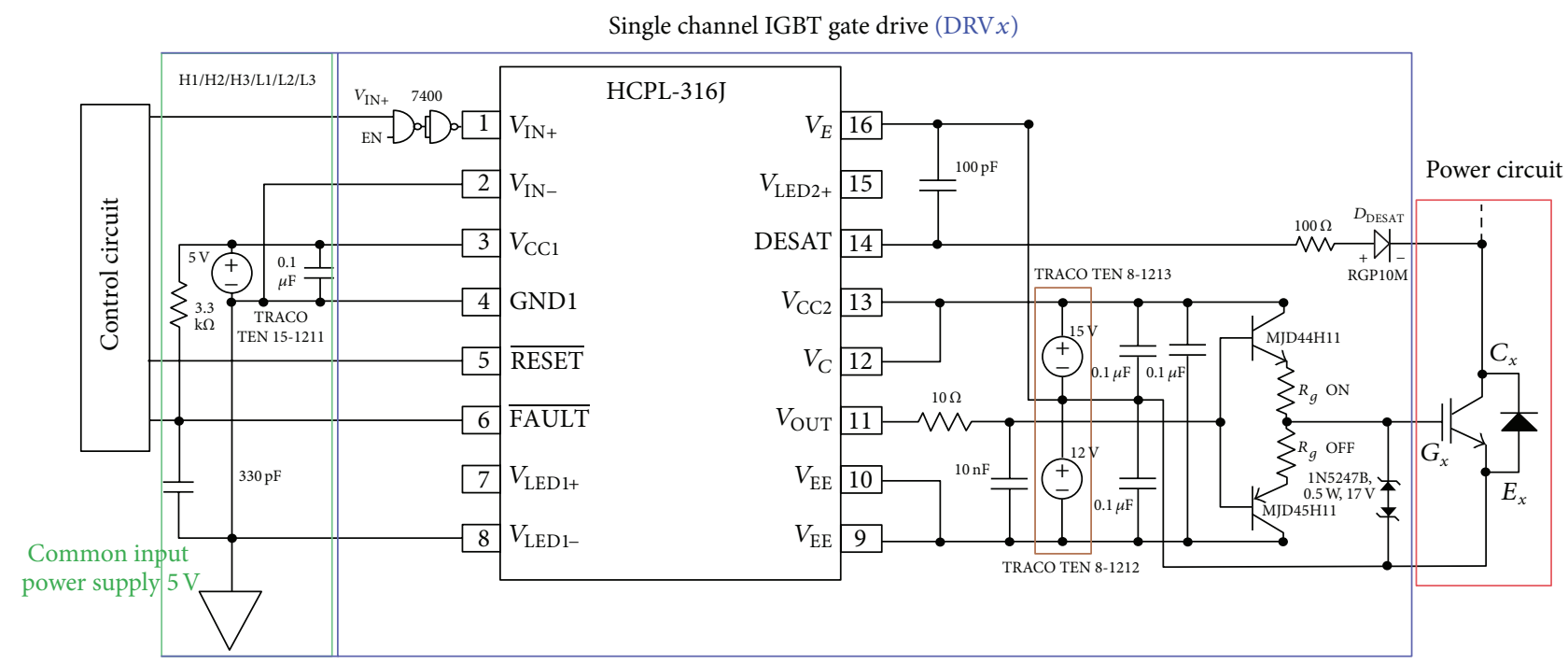

Figure 2: IGBT gate driver circuit using HCPL-316J.

The higher gate current and negative drive requirements of the IGBTs are addressed by designing a totem pole arrangement using matched NPN-PNP transistors as shown in Figure 2. The figure shows matched NPN and PNP transistors to provide push pull IGBT gate drive current amplified, to satisfy the gate drive requirements. This ensures fast turning $\mathrm{ON}$ and turning OFF of the IGBT. While boosting the output currents from IC Drivers, shoot through phenomenon can be avoided in this case. The pair of transistors protects each other's base-emitter junctions and handles current surges quite well. Complete isolation of the digital and power stages is ensured using this driver circuit to facilitate a fully safe operation of the digital controller even during the high voltage stresses in the output stage of the inverter.

2.2. Single Phase H-Bridge Power Stage. A single phase Hbridge inverter power stage is constructed to drive the electromagnet with sufficient amount of current as shown in Figure 3. The purely inductive nature of the load is not common for typical inverter applications and it can produce large voltage stress on the IGBT switches [9]. To avoid any type of overrated voltage spikes on the inverter switches, both snubber capacitors and varistors are connected in parallel to each switch.

2.3. Load Current Limit Circuit. The driver circuit ensures that the inverter bridge is protected against over loads and voltage stresses. The load should be also protected from over current to safe guard the electromagnet. This is achieved using a series current limiting circuit along with an accurate hall effect current sensor as shown in Figure 4. This allows the user to set the current limits for the load and control the inverter output accordingly. If the specified limit is crossed, the series current limiting circuit detects a fault and shuts down the inverter operation.
2.4. Labview-FPGA Based Digital Controller. The complete control logic is implemented in an FPGA which is programmed using Labview as shown in Figure 5. The system is sufficiently fast and all the feedback signals can be incorporated into a single FPGA. This helps to sense the fault status output from the inverter driver and execute any tertiary control action in case of any fault condition in the output load.

\section{Simulation and Prototype Development}

The aim of this project was to employ the power converter topology, described in Section 2, in the experimental setup which generates the alternating magnetic field in an air gap for testing the magnetocaloric properties of the materials. A solution with combined ac and dc field has been investigated and proposed. In this way, the magnetocaloric material does not need to be rotated or moved in and out of the air gap since the magnetic field is time-varying. It was of interest to obtain the field whose intensity and frequency can be easily changed, which is not the case with a pure PM machine, commonly used for the testing and characterizing of the magnetocaloric materials.

The solution with the combined ac and dc field can be realized by a three legged iron core, having the coil in one leg (creating the ac field), air gap in the middle leg, and permanent magnet in the third leg (creating the dc field). The alternating field in the air gap should be the superposition of the two fields from the other legs and positively biased, which is achieved by using permanent magnets creating a dc field. In this way, both the effects from both ac and dc field could be separately studied. The biased magnetic field should be achieved in the case when the two fields (ac and dc) are contributing in the same direction to the field in the air gap. The frequency of the magnetic field should be variable $(0-50 \mathrm{~Hz})$, to enable investigation of how magnetocaloric materials react in different time scales. 


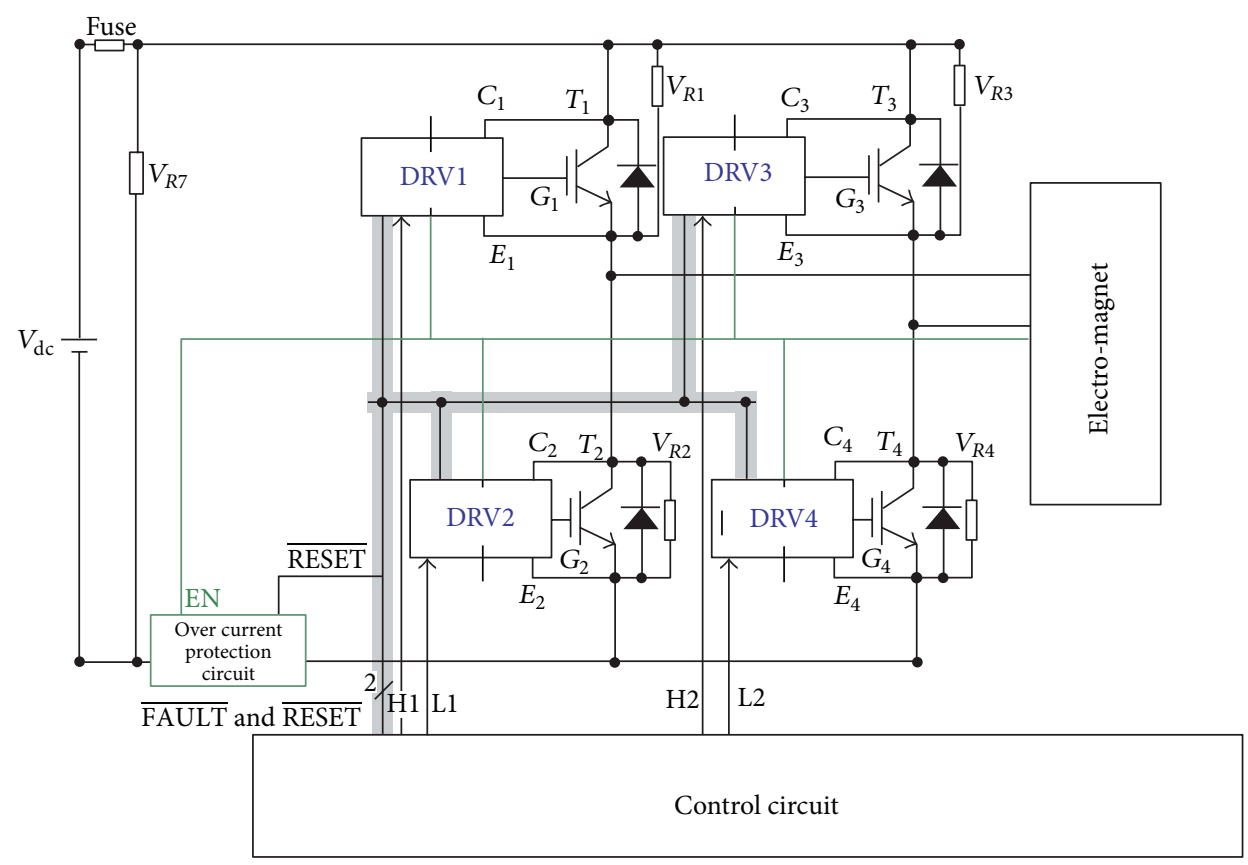

FIgURE 3: Single phase H-bridge inverter power stage.

Over current protection circuit

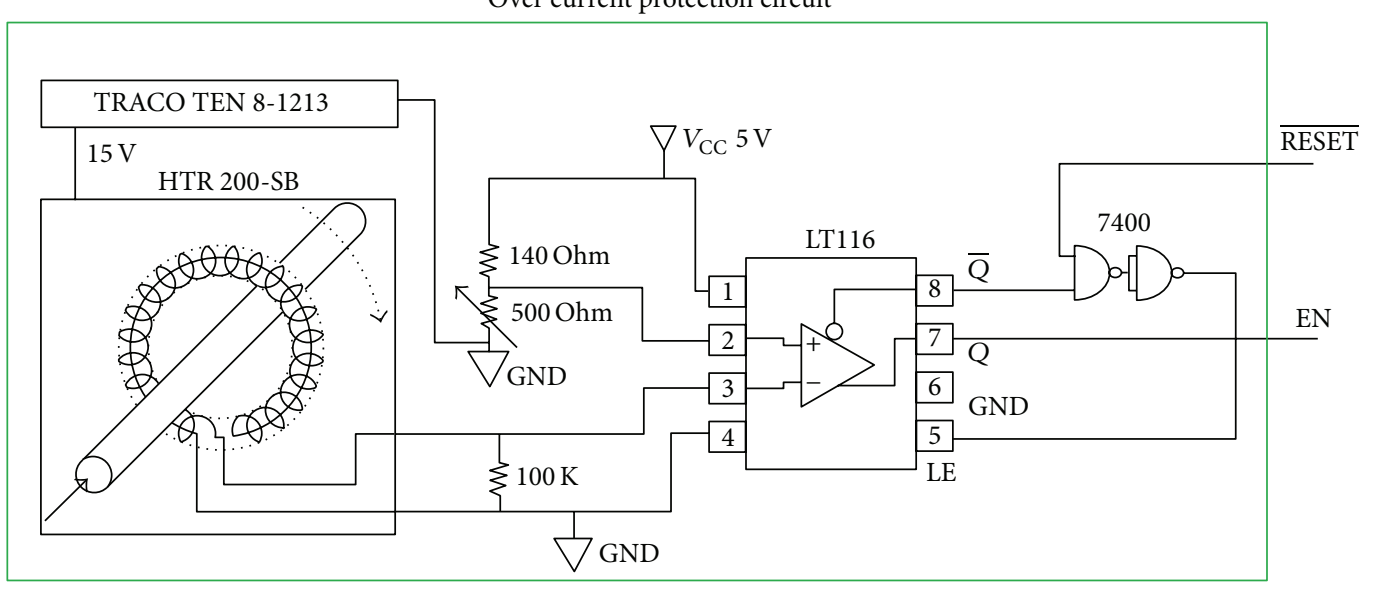

FIgURE 4: Load current limiting and protection circuit.

For the purpose of the initial experimental verifications, a prototype design of the three legged iron core has been proposed, having the dimensions and magnetic properties according to Figures 6 and 7. The coil leg is supposed to be slightly thinner than other legs, in order to reach saturation in that leg, forcing the dc field through the air gap.

To calculate the area $A$ corresponding to a specific magnetic flux, $\Phi$, and magnetic flux density, $B$, the following equation was used:

$$
\Phi=\int_{A} B \cdot d A .
$$

The number of ampere-turns needed in the coil to generate the desired magnetic flux density was found using the formula bellow, neglecting the leakage flux:

$$
N I=H_{g} l_{g}+H_{c} l_{c}
$$

where $H_{g}$ is the magnetic field intensity in the air gap $\left(B_{g} / \mu_{0}\right)$, $l_{g}$ is the length of the air gap, $H_{c}$ is the magnetic field intensity in the core $\left(H_{c}=H_{g}\right)$, and $l_{c}$ is the length of the core. NI was found to be approximately 1970 AT (leakage is neglected), which can be realized with the coil having 786 turns and the current of $2.5 \mathrm{~A}$, being reasonable and under the limit of the protection circuit.

The data of the proposed iron core are introduced in Comsol simulation tool. The first leg of the iron core is 


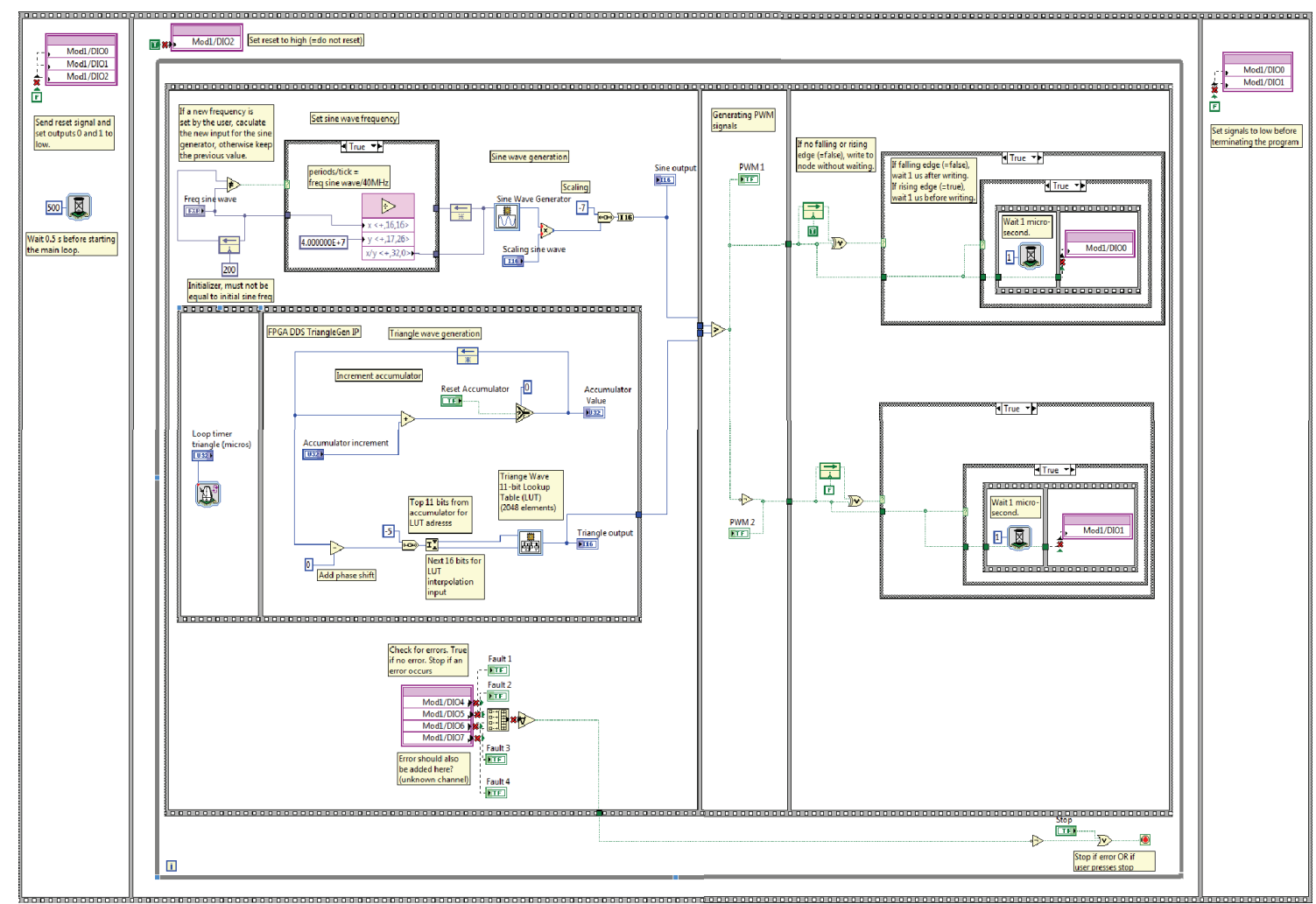

FIgURE 5: Control loop in Labview.

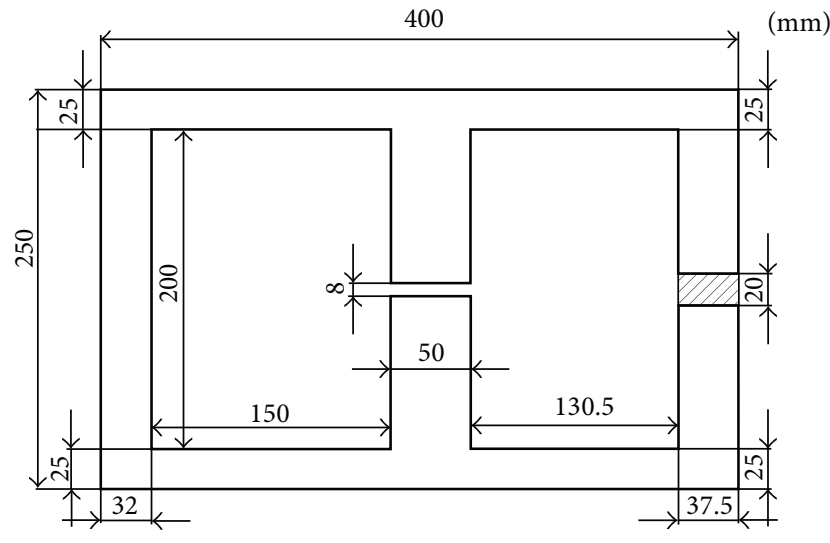

FIgURE 6: The dimensions of the three legged iron core prototype.

excited by the coil having 786 turns, which was estimated to be sufficient with rather low currents. In the middle leg of the core there is the air gap of $8 \mathrm{~mm}$, as the testing area of the magnetocaloric materials. The third leg of the core is excited by the permanent magnet with a remanence of $1.3 \mathrm{~T}$. By changing the ampere-turns of the first leg, the different distributions of the field are investigated in the air gap leg. Of interest was to find the current for which the two fields (ac and $\mathrm{dc}$ ) are contributing the same. The current of $3 \mathrm{~A}$ (close to approximated $2.5 \mathrm{~A}$ ) was found to be the appropriate one, in the case when having 786 turns.

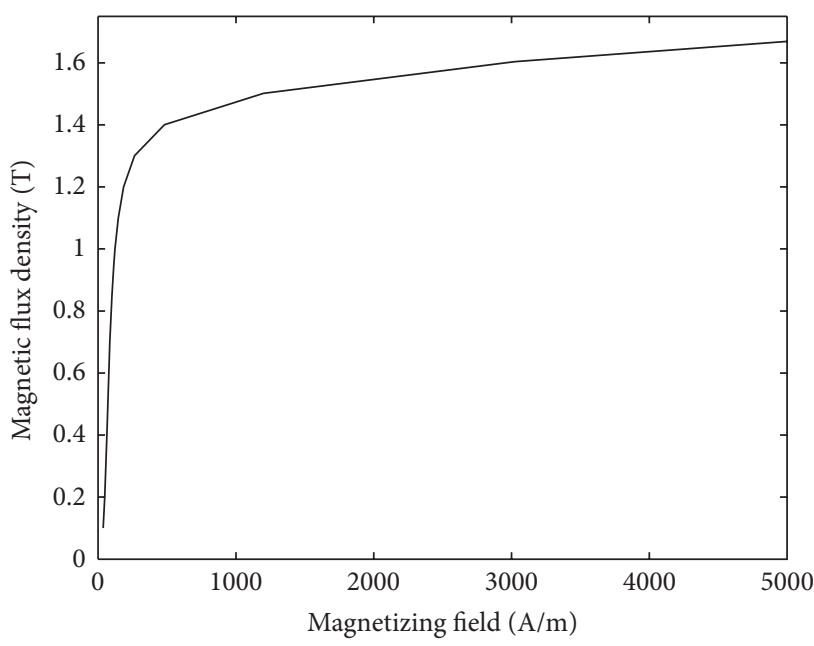

Figure 7: The magnetic properties of the three legged iron core prototype.

In Figure 8 the static simulation results for the case when there is the equal contribution of the two fields in the leg with the air gap are shown, having the dc current of $3 \mathrm{~A}$ and reaching the averaged field in the air gap $B=0.28 \mathrm{~T}$.

\section{Experimental Results}

The three legged iron core proposed and studied in the previous section has been built. The iron core consists of 


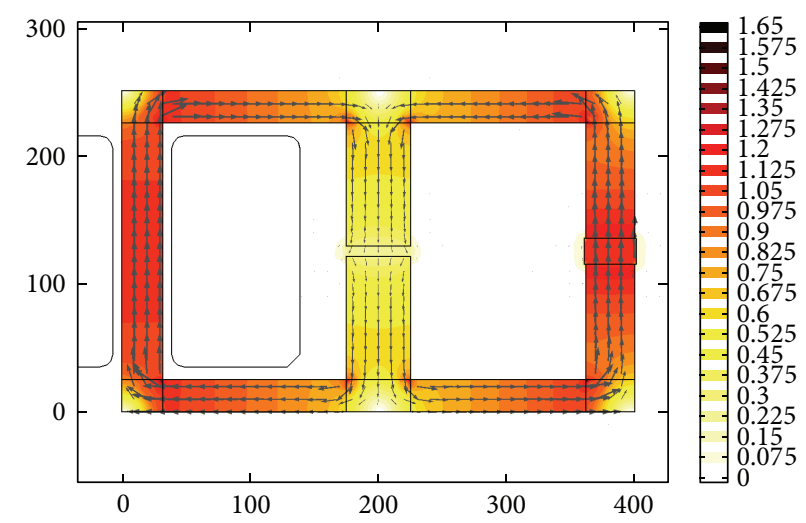

FIgURE 8: Static simulation results of the flux density distribution in the three legged iron core in the case of $3 \times 786$ ampere-turns.

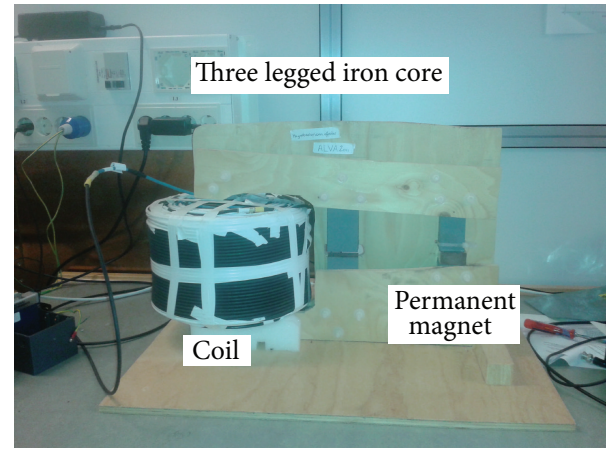

(a)

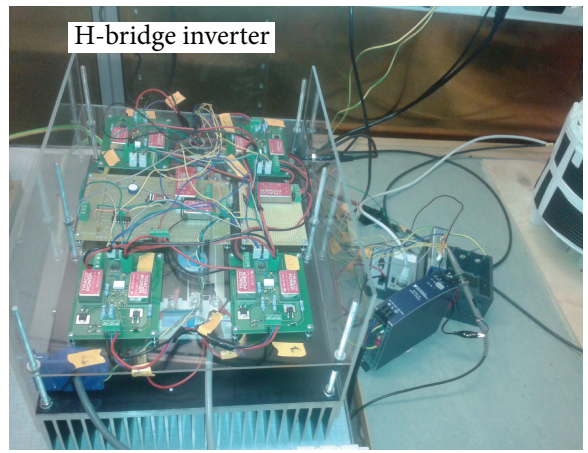

(b)

FIGURE 9: Experimental setup of the iron core (a) and power electronics (b).

$0.5 \mathrm{~mm}$ thick sheets of silicon steel M350-50A with C6 isolation coating (organic based coating), having the dimensions and magnetic properties according to Figures 6 and 7. The core was laminated to decrease the eddy currents. The sheets were stacked into a support structure and compressed using polyamide threaded rods. The coil having 786 turns is attached to the first leg of the core, with the cablecross section of $6 \mathrm{~mm}^{2}$. The air gap of $8 \mathrm{~mm}$ is placed in the middle leg of the iron core, that is, the area for the testing of magnetocaloric materials. Neodymium iron boron permanent magnet with dimensions $2 \mathrm{~cm} \times 4 \mathrm{~cm} \times 4 \mathrm{~cm}$ (magnet grade N42) and a remanence of $1.3 \mathrm{~T}$ is mounted in the third leg. The three legged iron core is shown on Figure 9(a).

The coil in the first leg of the iron core is supplied by Hbridge inverter, as described in Section 2. The inverter with drive circuit is shown in Figure 9(b). The real-time implementation of the algorithm was made by FPGA using a Labview/CompactRio real-time module, which is plugged into a PC. The experiments have been done by feeding a coil with an inductance of $L=0.36 \mathrm{H}$. The total dc voltage provided from the external dc supply $360 \mathrm{~V} / 15 \mathrm{~A}$ was $80 \mathrm{~V}$. Switching frequency was set to $1 \mathrm{kHz}$ and fundamental frequency was $5 \mathrm{~Hz}$. No filters were applied in pre- or postprocessing of the results. The results of the experimental verification are shown in Figure 10. In Figure 10(a) the current in the coil $i_{L}(t)$ is shown, having a maximum of $\approx 3 \mathrm{~A}$. The current is obviously distorted, due to the magnetic saturation in the part of the core close to the coil leg and due to the effect of the bias introduced by the permanent magnet. The magnetic flux density is shown in the Figure 10(b), having the peak value $B$ very close to the simulated $0.28 \mathrm{~T}$ and it is measured in the air gap with a hall effect magnetometer. The peak power consumed from the supply in this case was $184 \mathrm{~W}$.

The peak values from of the coil current and the field in the air gap in Figure 10 show the good agreement with the simulation done in Comsol for a static $I-B$ pairs. The measured field in the air gap is slightly higher than the simulated one, which can be caused by the current being slightly higher and having the averaged field across the air gap in the simulation.

However, for testing the magnetocaloric effect and for the magnetocaloric applications, having a large adiabatic temperature change, $\Delta T_{\mathrm{ad}}$, and reaching high entropy change, $\Delta S$, are equally important. One has to optimize simultaneously since the two are not directly coupled. To be really of interest for the magnetocaloric applications, the large $\Delta T_{\text {ad }}$ and $\Delta S$ should be reached for the intermediate field changes, that is, preferably $\Delta B \leq 1.4 \mathrm{~T}$. Accordingly, most of the studies are focused on the fields $B \approx 1 \mathrm{~T}$. For this reason, the preliminary testing system presented in this paper needs to be scaled up, to be able to reach the biased alternating field 0-1 T. In terms 


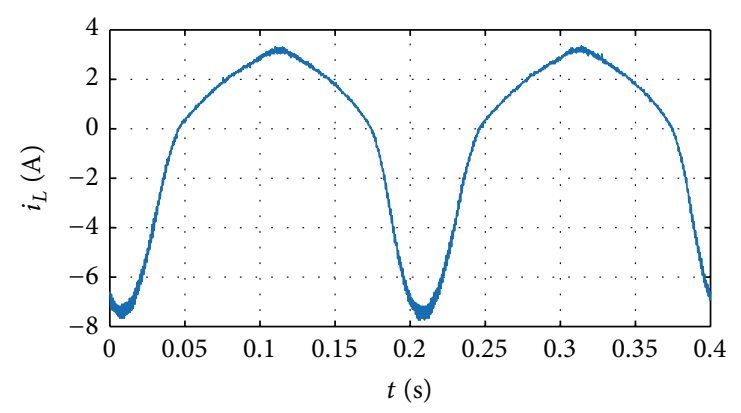

(a)

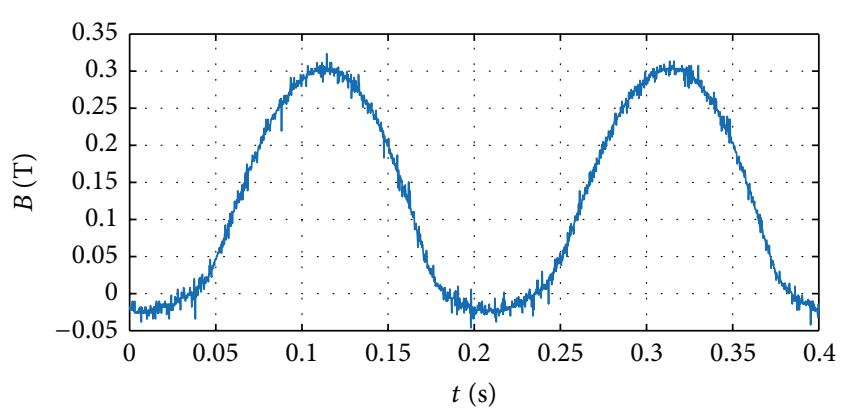

(b)

FIGURE 10: Experimental results: (a) load current, (b) measured flux density in the air gap.

of the three legged core dimension limitations, the maximum field in the air gap obtained for this setup was $0.4 \mathrm{~T}$, for a highly distorted current having a maximum $\approx 30 \mathrm{~A}$. For this measurement the effect of the ac field was dominated, and the resulting field in the air gap is not properly biased. With more robust magnetic core it is possible to reach the desired field in the air gap, without entering in the saturation in the coil leg.

\section{Conclusion}

A new and improved method of characterizing magnetocaloric materials using a power converter based test platform is presented in this work. It helps to eliminate the limitations of conventional test methods using magnetic machines with moving parts and adds a lot of flexibility in the control of flux density and frequency. In particular, the method is based on combining the rather conventional power electronics with essential control and additional protections, and the novel idea to employ a specific magnetic circuit which can produce biased alternating magnetic field in the air gap, for the testing of the magnetocaloric materials.

The simulation and experimental results are presented to validate the new test platform performance. The proposed prototype has shown the match performance between the simulations done for static $I-B$ pairs and the experiments. Alternating and properly biased magnetic flux density was measured in the air gap, having the maximum very close to the expected value.

The new method explores the complete advantages of power electronic based excitation compared to the machine based one and provides improved overall performance. Since the scaled down prototype showed the expected performance, a new prototype with the improved iron core able to reach the required intermediate fields, that is, $B \approx 1 \mathrm{~T}$, needs to be built and tested.

\section{Conflict of Interests}

The authors declare that there is no conflict of interests regarding the publication of this paper.

\section{Acknowledgments}

This study was carried out with funding from the Swedish Research Council, Grant no. 2012-4706. The authors would like to thank the KIC InnoEnergy Ph.D. school for providing all the added value educational activities. Thanks are due to SweGRIDS, EIT KIC InnoEnergy, Swedish Energy Agency, and STandUP for Energy for their support.

\section{References}

[1] K. A. Gschneidner Jr. and V. K. Pecharsky, "Thirty years of near room temperature magnetic cooling: where we are today and future prospects," International Journal of Refrigeration, vol. 31, no. 6, pp. 945-961, 2008.

[2] H. Yibole, F. Guilliou, L. Zhang, N. H. van Dijk, and E. Brück, "Direct measurement of the magnetocaloric effect in $\mathrm{MnFe}(\mathrm{P}, \mathrm{X})(\mathrm{X}=\mathrm{As}, \mathrm{Ge}, \mathrm{Si})$ materials," Journal of Physics D: Applied Physics, vol. 47, no. 7, Article ID 075002, 2014.

[3] B. Yu, M. Liu, P. W. Egolf, and A. Kitanovski, "A review of magnetic refrigerator and heat pump prototypes built before the year 2010," International Journal of Refrigeration, vol. 33, no. 6, pp. 1029-1060, 2010.

[4] V. K. Pecharsky, K. A. Gschneidner Jr., Y. Mudryk, and D. Paudyal, "Making the most of the magnetic and lattice entropy changes," Journal of Magnetism and Magnetic Materials, vol. 321, no. 21, pp. 3541-3547, 2009.

[5] O. Gutfleisch, M. A. Willard, E. Brück, C. H. Chen, S. G. Sankar, and J. P. Liu, "Magnetic materials and devices for the 21st century: stronger, lighter, and more energy efficient," Advanced Materials, vol. 23, no. 7, pp. 821-842, 2011.

[6] A. Kitanovski and P. W. Egolf, "Innovative ideas for future research on magnetocaloric technologies," International Journal of Refrigeration, vol. 33, no. 3, pp. 449-464, 2010.

[7] R. Bjørk, C. R. H. Bahl, A. Smith, and N. Pryds, "Review and comparison of magnet designs for magnetic refrigeration," International Journal of Refrigeration, vol. 33, no. 3, pp. 437-448, 2010.

[8] Avago Technologies, "2.5 Amp Gate Drive Optocoupler with Integrated (VCE) Desaturation Detection and Fault Status Feedback," HCPL-316J datasheet, April 2009.

[9] N. Mohan, T. M. Undeland, and W. P. Robbins, Power Electronics: Converters, Applications, and Design, John Wiley \& Sons, New York, NY, USA, 3rd edition, 2003. 

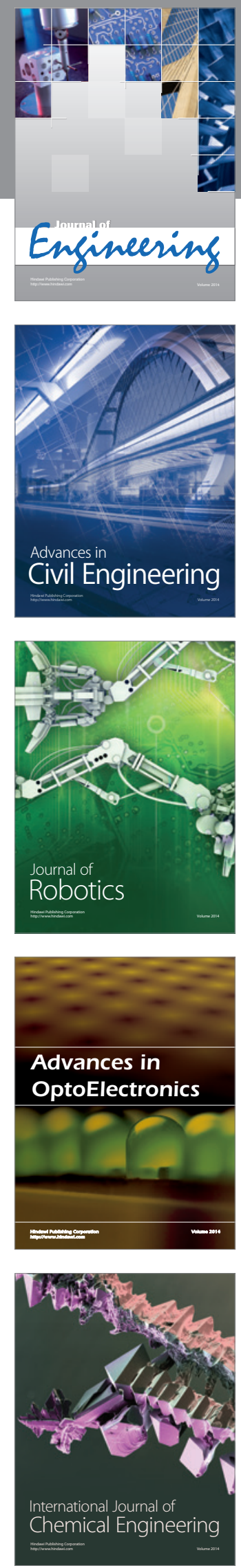

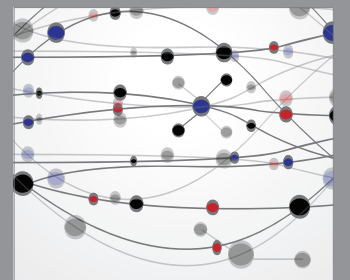

The Scientific World Journal
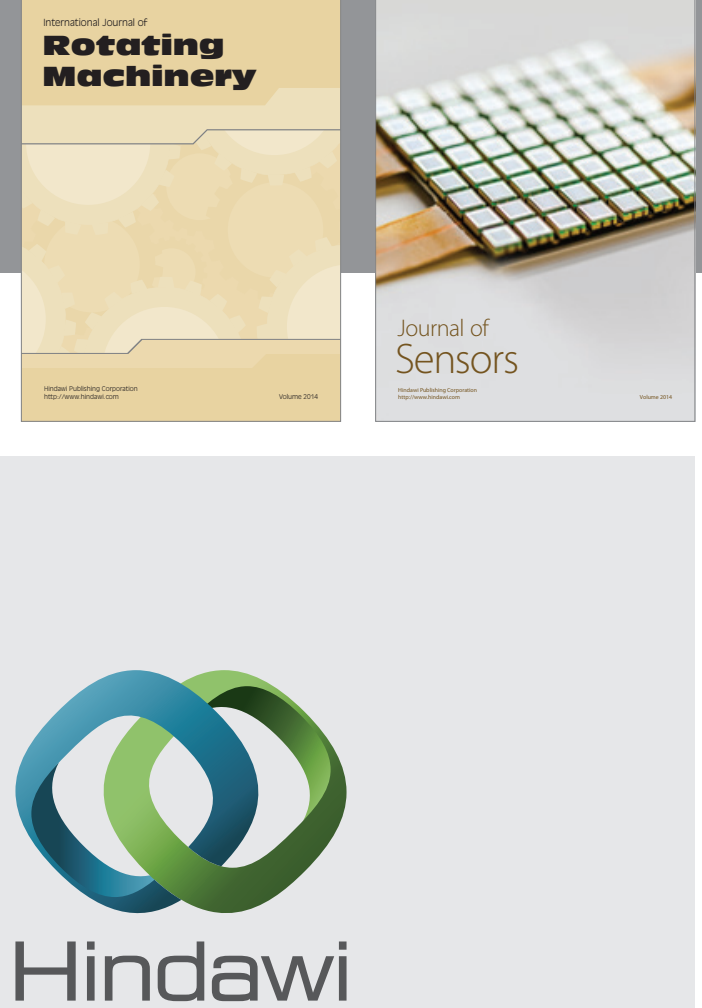

Submit your manuscripts at http://www.hindawi.com
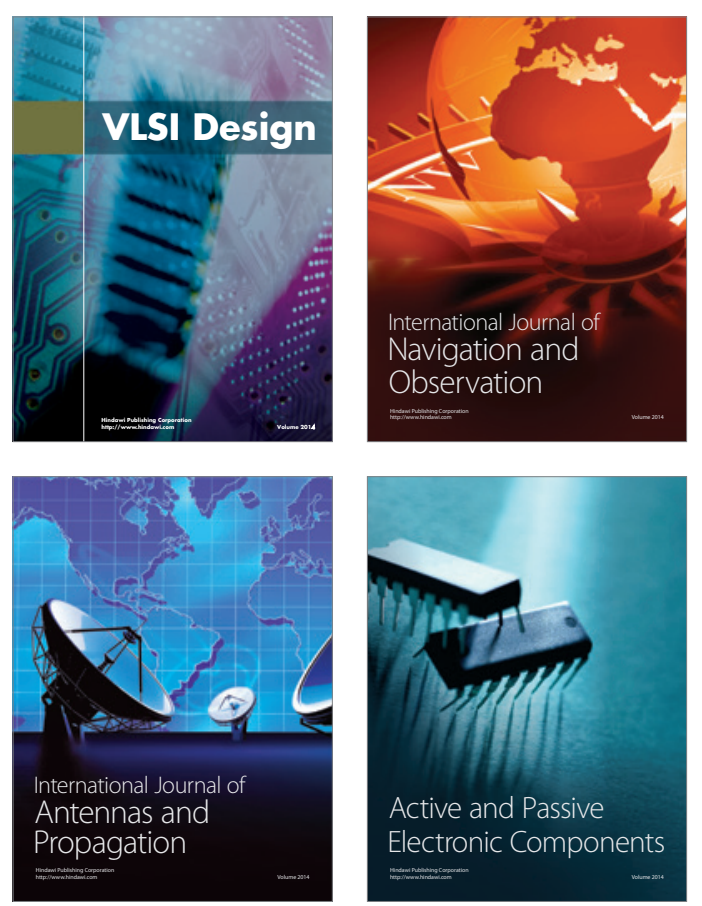
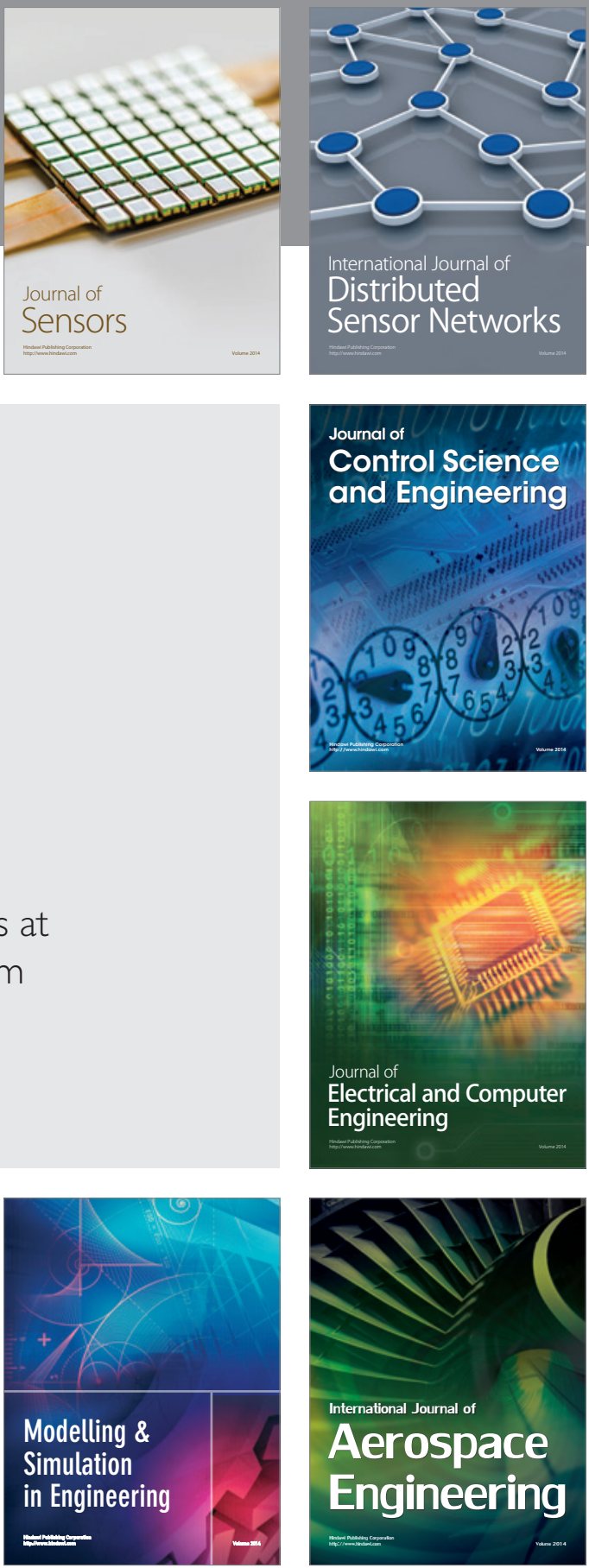

Journal of

Control Science

and Engineering
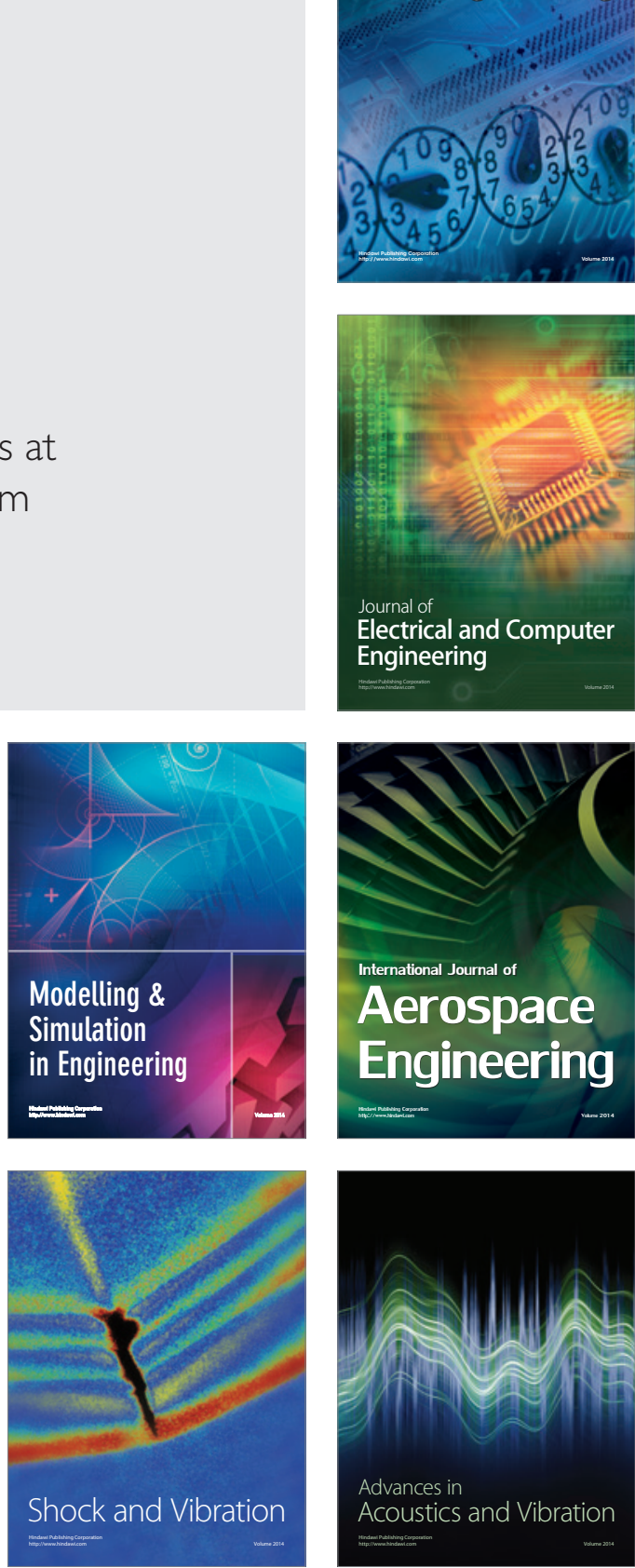